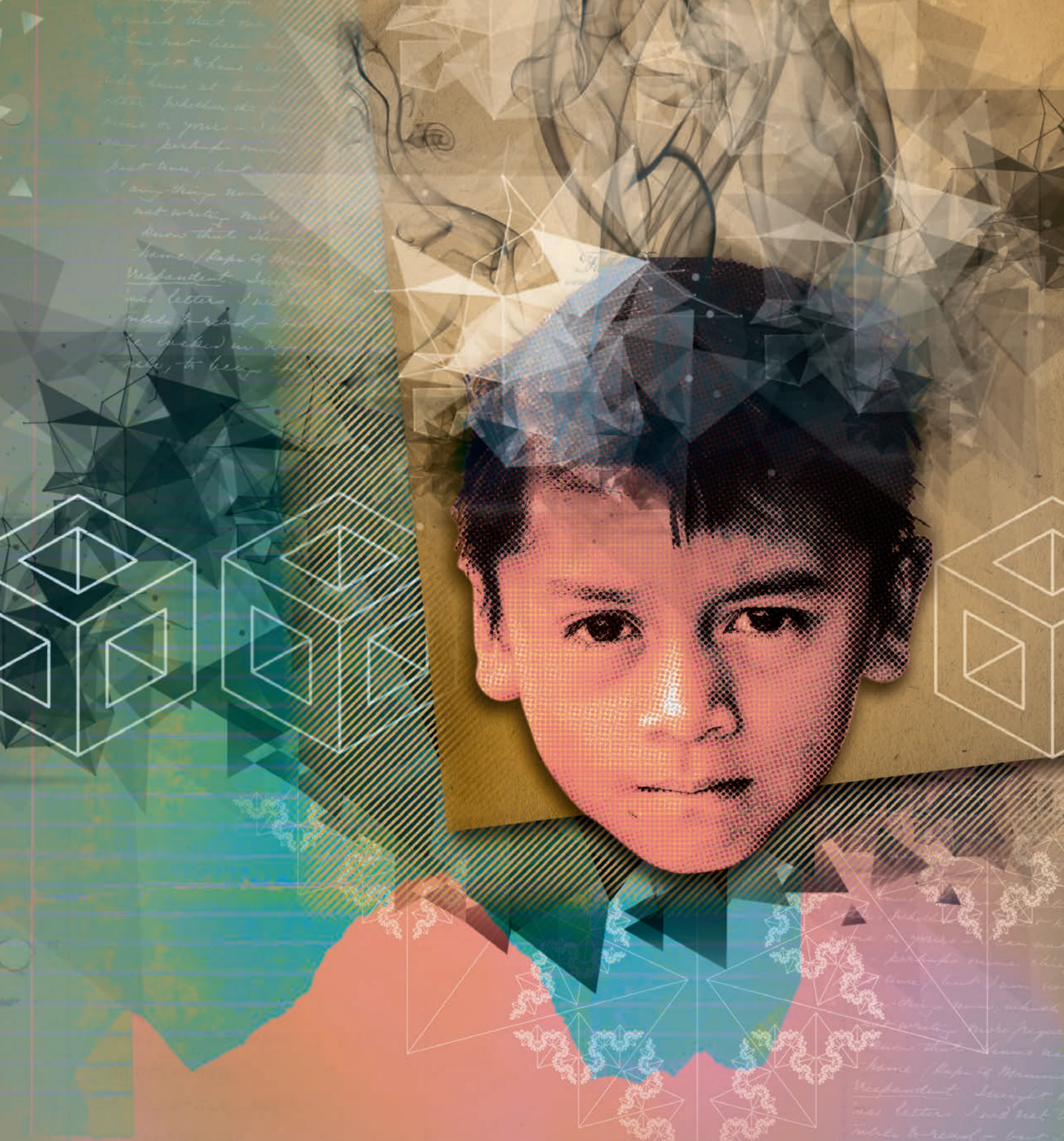




\section{DESARROLLO DE LAS COMPETENCIAS MATEMÁTICAS EN EL PENSAMIENTO \\ GEOMÉTRICO, A TRAVÉS DEL MÉTODO HEURÍSTICO DE POLYA

\section{Development of mathematical competences in geometric thinking, through Polya's heuristic method}
Desenvolvimento das competências matemáticas no pensamento geométrico, através do método heurístico de Polya

\section{RECIBIDO:15 DE OCTUBREDE 2017}

Eliana Sáenz Mass (Colombia)

eliesamass@gmail.com

Magíster en Educación

Secretaria de Educación

Municipal (Montería)

\section{es}

\section{RESUMEN}

Este artículo presenta los resultados de la investigación cuyo propósito fue evaluar la eficacia del método heurístico de Polya (1981), en el desarrollo de las competencias matemáticas en el pensamiento espacial. Esta investigación se desarrolló desde el enfoque cuantitativo con diseño cuasi-experimental; se utilizó un test para identificar los desempeños en las competencias de razonamiento, resolución de problemas y comunicación en el pensamiento geométrico en dos grupos de quinto grado de la institución educativa Villa Cielo, del municipio de Montería (CórdobaColombia), antes y después de la intervención. Se aplicó una estrategia didáctica en el tema de los sólidos geométricos, teniendo en cuenta los pasos de la resolución de problemas desde el modelo de Polya y la estrategia de trabajo cooperativo. Los resultados obtenidos se analizaron mediante prueba estadística T- student. Se evidenció que los estudiantes, después de la intervención, mejoraron significativamente en los desempeños de las competencias, corroborando así la eficacia de la estrategia.

PALABRAS CLAVE: pensamiento geométrico, resolución de problemas, método heurístico de Polya, competencias

\section{EVALUADO:7DENOVIEMBRE DE 2017}

María Patiño Garcés (Colombia) marypatygar@gmail.com Magíster en Educación Secretaria de Educación Municipal (Montería)

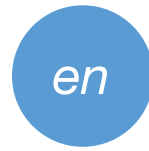

\section{ABSTRACT}

This article presents the research results, whose purpose was to evaluate the effectiveness of Polya heuristic method (1981), in spatial thinking mathematical competences development. This research was developed from a quantitative approach with quasi-experimental design; a test was used to identify the performance in reasoning skills, problem solving and communication in geometric thinking in two fifth grade student's groups of Villa Cielo educational institution, municipality of Monteria (Córdoba-Colombia), before and after the intervention. A didactic strategy was applied in geometric solids matter, considering the Polya model steps of solving problems, and the cooperative work strategy. Obtained results were analyzed by means of statistical T-student test. It was evidenced that students, after the intervention, improved significantly in competencies performance, corroborating the strategy effectiveness.

KEYWORDS: geometric thinking, problem solving, Polya heuristic method, competencies
ACEPTADO:27 DE NOVIEMBREDE 2017

Juana Robles González (Colombia) juanarobles2003@yahoo.com Magister en Estadística Universidad de Córdoba

\section{por}

\section{RESUMO}

Este artigo apresenta os resultados da pesquisa cujo propósito foi avaliar a eficácia do método heurístico de Polya (1981), no desenvolvimento das competências matemáticas no pensamento espacial. Esta pesquisa foi desenvolvida desde o enfoque quantitativo com desenho quase-experimental; utilizou-se um teste para identificar os desempenhos nas competências de razoamento, resolução de problemas e comunicação no pensamento geométrico em dois grupos da quinta série da Instituição educativa Villa Cielo, do município de Montería (Córdoba-Colômbia), antes e depois da intervenção. Aplicou-se uma estratégia didática no tema dos sólidos geométricos, tendo em conta os passos da resolução de problemas desde o modelo de Polya e a estratégia de trabalho cooperativo. Os resultados obtidos analisaram-se mediante teste estatístico T - student. Se evidenciou que os estudantes, depois da intervenção, melhoraram significativamente nos desempenhos das competências, corroborando assim a eficácia da estratégia.

PALAVRAS CHAVE: pensamento geométrico, resolução de problemas, método heurístico de Polya, competências

\section{PARA CITAR ESTE ARTíCULO/TO CITE THIS ARTICLE/PARA CITAR ESTE ARTIGO:}

Sáenz, E., Patiño, M.y Robles, J. (2017). Desarrollo de las competencias matemáticas en el pensamiento geométrico, a través del método heurístico de Polya. Panorama, 11(21),53-67. 


\section{INTRODUCCIÓN}

El pensamiento geométrico es uno de los componentes

María Patiño

Garcés |

Eliana Sáenz Mass |

Juana Robles González |

Panorama pp. 53-67|

Volumen 11 |

Número 21 | Julio-Diciembre |

2017 |

Por otra parte, los resultados de las pruebas en el municipio de Montería y la institución educativa Villa Cielo son similares a los obtenidos a nivel nacional, el 79\% y $91 \%$ respectivamente, se encuentran discriminados en el nivel mínimo e insuficiente, lo cual indica que los estudiantes no solucionan situaciones problema de menor complejidad.

Hay que mencionar, además, que los bajos desempeños de los estudiantes, las debilidades presentadas en las competencias y el componente geométrico especialmente son referentes para tomar estas como objeto de estudio en la investigación. Es conveniente subrayar que investigadores tales como Báez e Iglesias (2007) y Paredes et al. (2007), expresan que: la enseñanza de la geometría en la mayoría de los establecimientos educativos se imparte de manera tradicional, caracterizado por el trabajo en grupos y el discurso del docente como principal medio didáctico, no se tiene en cuenta los Proyectos Educativos Institucionales y la enseñanza de ella se realiza a través del lápiz y papel, tablero y marcador, negándole la posibilidad al estudiante de desarrollar su propia creatividad en busca de aprendizajes duraderos $\mathrm{y}$ eficaces.

Además, Hernández y Villalba (2001) afirman que, si al estudiante se le presenta la geometría como un producto final y terminado, se le niega la posibilidad de fomentar la creatividad, desarrollar habilidades y competencias que propicien aprendizajes significativos. En este sentido, la estrategia didáctica propuesta cobra gran importancia en el desarrollo de las competencias matemáticas a partir del método heurístico de Polya (1981).

En esta investigación se estableció como objetivo general evaluar la eficacia del método heurístico de Polya (1981), para el desarrollo de las competencias matemáticas del pensamiento geométrico, en estudiantes de quinto grado de la institución educativa Villa Cielo.

Teniendo en cuenta el objetivo general de esta investigación, a continuación se presenta el origen, la definición y las características de la resolución de problemas, sus pasos, las habilidades del pensamiento geométrico y el trabajo cooperativo.

\section{MARCO CONCEPTUAL}

RESOLUCIÓN DE PROBLEMAS EN LA ENSEÑANZA DEL PENSAMIENTO GEOMÉTRICO

Estudios relacionados con la enseñanza de las matemáticas muestran a la resolución de problemas como una 
estrategia o herramienta por medio de la cual los estudiantes construyen su propio conocimiento. Debido a esto, se sugiere que la enseñanza de la geometría gire en torno a la resolución de problemas donde se involucre el uso de relaciones y conceptos geométricos (Peña, 2008).

Para Polya (1981), la resolución de problemas es un método que permite al estudiante utilizar diferentes heurísticas para solucionar un problema. La palabra heurística proviene del griego heuriskein, que significa descubrir, encontrar. Un método heurístico es un conjunto de estrategias para solucionar problemas y reglas de decisión utilizadas por los solucionadores de problemas, basada en la experiencia previa.

Según Peralta (2000), el método heurístico es una actividad que contribuye a la formación de estudiantes activos, constructores de su propio aprendizaje. Por ejemplo, en geometría no es el estudio de las figuras, sino de las propiedades que permanecen invariantes respecto a ciertos tipos de transformaciones prácticas.

Para Polya, en su libro "Cómo plantear y resolver problemas", el término "heurística" lo describe como el arte de la resolución de problemas. La heurística trata de comprender el método que conduce a la solución de problemas, en particular las operaciones mentales típicamente útiles en este proceso (Polya, 1965, p. 102). Su método se sintetiza en cuatro pasos:

Tabla 1. Pasos del método heurístico de Polya

\section{PASOS DEL MODELO DE GEORGE POLYA}

Lee el problema detenidamente, lo expresa con sus propias palabras, identifica los datos del problema, discriminando la información necesaria, realiza esquemas o gráficos.

Plantean diferentes estrategias para solucionar el problema, entre ellas, buscar semejanzas con Configurar un plan otros problemas, enunciar el problema de otra manera, busca diferentes heurísticas para su solución.
Se implementa las estrategias seleccionadas,

Ejecutar el plan se revisa lo adecuado de la estrategia para solucionar la situación y utilizar nuevas dado el caso.

Se dan razones de la solución de la situación planteada.

Mirar hacia atrás

Se comparan diferentes estrategias de solución

Análisis de las estrategias para solucionar otros problemas

\section{COMPETENCIAS MATEMÁTICAS}

Según el proyecto PISA de la OCDE (2015), define la competencia como:

Capacidad de un individuo para identificar y comprender el papel que las matemáticas juegan en el mundo, realizar razonamientos bien fundados y utilizar e involucrarse en las matemáticas de manera que satisfagan las necesidades de la vida del individuo como ciudadano constructivo, comprometido y reflexivo ( $\mathrm{p}$ 12).

Por otro lado, Tobón (2006), considera que las competencias son procesos complejos de desempeño con idoneidad en un determinado contexto, con responsabilidad. Adicionalmente, el MEN (2003), a través de los estándares básicos de competencia afirma que: "ser matemáticamente competente está relacionado con el saber qué, el saber qué hacer y el saber cómo, cuándo y por qué hacerlo", por tanto esto implica que las competencias están relacionadas al hacer, como al comprender que se hace y por qué se hace y de la actitud y disposición para realizar la actividad.

En el proceso de relacionar los conceptos matemáticos con situaciones del contexto,juegan un papel importante las competencias matemáticas como ejes generadores del conocimiento. Estas competencias el MEN (2006) las clasifica en cinco procesos, a saber: 1. comunicación, 2. razonamiento, 3 , modelación, 4 . la formulación, tratamiento y resolución de problemas, 5. la formulación, comparación y ejercitación de procedimientos. Para efectos de la investigación, estas competencias se definen de acuerdo con la clasificación del ICFES (2007).
Desarrollo de las competencias matemáticas en el pensamiento geométrico, a través del método heurístico de Polya
| Panorama | pp. 53-67 | Volumen 11 | Número 21 | Julio-Diciembre | 2017 
Comunicación y representación: según el MEN

(2006), esta competencia es:

La adquisición y dominio de los lenguajes

María Patiño

Garcés |

Eliana Sáenz

Mass |

Juana Robles

González |

\section{Gonzàlez} propios de las matemáticas ha de ser un proceso deliberado y cuidadoso que posibilite y fomente la discusión frecuente y explícita sobre situaciones, sentidos, conceptos y simbolizaciones; para tomar conciencia de las conexiones entre ellos y para propiciar el trabajo colectivo, en el que los estudiantes compartan el significado de las palabras, frases, gráficos y símbolos, aprecien la necesidad de tener acuerdos colectivos y aun universales, y valoren la eficiencia, eficacia y economía de los lenguajes matemáticos (p. 54).

Modelación, planteamiento y resolución de problemas: esta competencia permite entender situaciones problemas, desarrollar, aplicar y justificar las diversas estrategias utilizadas para solucionar las situaciones planteadas (ICFES, 2013).

Razonamiento y argumentación: el razonamiento es el conjunto de habilidades, conocimientos y actitudes relacionado con la explicación de los procesos realizados para la solución de un problema (Tobón, 2007).

\section{TRABAjo CoOperativo}

Para Ferreiro y Calderón (2001), el aprendizaje cooperativo es un modelo educativo innovador que propone una manera distinta de organizar la educación escolar a diferentes niveles: de escuela en su totalidad, en tal sentido es un modelo de organización institucional; del salón de clases, siendo entonces una forma de organización de la enseñanza y el aprendizaje; pero también puede ser considerado como un método o técnica para aprender.

Panorama |

pp. 53-67|

Volumen 11।

Número 21 |

Julio-Diciembre |

2017 |

Por otro lado, Pujolás (2009), define el aprendizaje cooperativo como:

El uso didáctico de equipos reducido de estudiantes, generalmente de composición heterogénea, en rendimiento y capacidad, aunque ocasionalmente puede ser más homogéneo, utilizando una estructura de la actividad, tal que se asegure al máximo la participación igualitaria y se potencie al máximo la interacción simultanea $56 \mid$

\section{Metodología}

Analizando las características de la investigación, y teniendo en cuenta que la asignación de los grupos no fue aleatoria, se eligió un diseño cuasi experimental con el que se pretende evaluar el impacto de los tratamientos y/o procesos de cambios en situaciones donde los sujetos objetos de estudio no han sido asignados de manera aleatoria (Arnau, 1995).

Por lo tanto, se tomaron dos grupos (control y experimental), donde al grupo experimental se intervino con estrategias didácticas mediadas por el método heurístico de Polya mediante la metodología de trabajo cooperativo, con el propósito de desarrollar las competencias matemáticas; se les aplicó pretest y postest (ver Anexo 1).

La población objeto de estudio son los estudiantes de quinto grado de la institución educativa Villa Cielo, que tiene tres grupos en la jornada de la tarde, para un total de 102 estudiantes. Para la elección del grupo experimental y control se realizó de manera aleatoria, conformado así: $5^{\circ} 1$ (Control) y $5^{\circ} 3$ (Experimental).

Por otro lado, atendiendo al objetivo general de esta investigación se identifican las siguientes variables:

La variable independiente en esta investigación es la intervención de una estrategia didáctica basada en el método heurístico de Polya (1981), en el pensamiento geométrico, en la unidad de estudio de los sólidos geométricos, utilizando la metodología de trabajo cooperativo, con el fin de desarrollar las competencias matemáticas (razonamiento, comunicación y resolución de problemas). Se planearon en cada una de las etapas de intervención distintas situaciones propias de las matemáticas y fuera de ellas.

Por otra parte, para la elaboración de las guías didácticas en la unidad de estudio, los sólidos geométricos, se tuvo en cuenta los Estándares Básicos de Competencias en Matemáticas (MEN, 2006, p. 82), Derechos Básicos de Aprendizajes (MEN, 2015), Matriz de Referencia de matemáticas (MEN, 2015), relacionados con el pensamiento geométrico. (Anexo 2). 
Nombre de la Sesión Propósito de Aprendizaje

\section{Indicadores de Competencias}

Comunicación: describir las formas tridimensionales según la forma y sus

Descubrir las caras y características

Huellas de los cuerpos geométricos de huellas dejadas por los cuerpos geométricos

características

Razonamiento: comparar y clasificar objetos tridimensionales y figuras bidimensionales de acuerdo con sus componentes

Resolución de Problemas: utilizar relaciones y propiedades geométricas para resolver problemas

\section{Comunicación:}

- Diferenciar los atributos mensurables de un objeto.

Comparando objetos del entorno y clasificando sólidos geométricos
Interpretar, comparar y justificar propiedades de formas bidimensionales y tridimensionales
Percepción visual de objetos
Representar gráficamente las diferentes vistas bidimensionales que tienen una forma tridimensional
Los sólidos en nuestro entorno.
- Identificar los atributos de un objeto que tienen la posibilidad de ser medido, longitud, superficie, espacio que ocupa.

\section{Razonamiento:}

- Comparar y clasificar objetos tridimensionales y figuras bidimensionales de acuerdo con sus componentes y propiedades.

- Resolución de Problemas

- Utilizar relaciones y propiedades geométricas para resolver problemas.

- Usar representaciones matemáticas en

el pensamiento geométrico,

a través

del método

heurístico de

Polya

Comunicación: diferenciar los atributos mensurables de un objeto

\section{Razonamiento:}

- Representar objetos tridimensionales a partir de representaciones bidimensionales.

- Resolución de Problemas:

- Utilizar relaciones y propiedades geométricas para resolver problemas

Comunicación: describir las formas tridimensionales según la forma y sus características.

Razonamiento: Identificar propiedades y características de sólidos.

Resolución de Problemas: resolver problemas que requieran identificar patrones y regularidades usando representaciones geométricas.

Comunicación: Describir procedimientos para la construcción de figuras y objetos dadas sus medidas

\section{Razonamiento:}

El arte de construir sólidos con material del medio
Construir y manipular figuras y representaciones gráficas el espacio

- Representar objetos tridimensionales a partir de representaciones bidimensionales

- Construir y descomponer figuras planas y sólidos a partir de condiciones dadas.

- Resolución de problemas:

- Usar representaciones geométricas y establecer relaciones entre ellas para solucionar problemas.

Comunicación:

- Interpretar información proveniente de situaciones prácticas de medición (armado de muebles, construcción de objetos).

- Describir procedimientos para la construcción de figuras y objetos dadas sus medidas.

Razonamiento

Relaciona objetos tridimensionales y sus propiedades con sus respectivos desarrollos planos
Desarrollo plano de los cuerpos geométricos.
- Relacionar objetos tridimensionales y sus propiedades con sus respectivos desarrollos planos

- Asociar desarrollos planos con los respectivos sólidos

- Resolución de problemas:

- Usar representaciones geométricas y establecer relaciones entre ellas para solucionar problemas.

- Resolver problemas que requieran identificar patrones y regularidades usando representaciones geométricas 
María Patiño

Garcés |

Eliana Sáenz Mass |

Juana Robles González |

Panorama pp. 53-67| Volumen 11 | Número 21 | Julio-Diciembre | 2017 | nes de trabajo, en el GE donde se aplica la estrategia, inicialmente se conformaron los grupos de trabajo cooperativos utilizando tres dinámicas (armar rompecabezas, dulces de colores y enumeración del 1 al 4), atendiendo a los niveles de intervención para trabajar cooperativamente, descrito por Pujolas (2009). En el
Por otro lado, para evitar el sesgo derivado del aprendizaje que se produce al realizar una prueba en repetidas ocasiones, se cambió el orden de las preguntas y respuestas en el postest.

En cuanto a la intervención en el GE, en primer lugar, las investigadoras socializaron a los estudiantes en qué consiste la intervención, cuándo y cómo se llevaría a cabo, quiénes la ofrecerían, cuál es el tema objeto de estudio y las competencias a desarrollarse.

En este mismo orden de ideas, al implementar las sesio- primer nivel, cohesionar el grupo, los estudiantes inician una etapa de conocimiento y cooperación entre ellos para llegar a un consenso; en el segundo, aprender trabajando en equipo, se hace rotación de los roles, pero se mantiene el mismo equipo de trabajo; por último, enseñar a trabajar en equipo, se realiza una rotación por grupos de expertos, en este caso los estudiantes se agrupan por roles comunes, comparten sus experiencias y retornan al grupo base a socializar lo aprendido.

Seguidamente, para el desarrollo de las actividades propuestas en las guías didácticas, se aplican los pasos propuestos por el modelo heurístico de Polya (Ver ejemplo en la tabla 3). En el grupo el primer paso (entender el problema), los estudiantes trabajan de manera individual, socializando con sus compañeros la comprensión de la situación problema, y así, en equipo realizan el segundo paso (configurar un plan); tercero ( ejecutar el plan) y cuarto paso (mirar hacia atrás); luego se construye colectivamente el concepto y se realiza el seguimiento al aprendizaje por medio de la participación, desarrollo y socialización de las actividades propuestas.

Tabla 3. Planteamiento de una situación problema

\section{Planteamiento de la Situación Problema}

¿Cómo son las caras que conforman y huellas de los cuerpos geométricos al plasmarse en una superficie plana? $¿$ Qué semejanzas y diferencias hay entre estas?

\begin{tabular}{|c|c|}
\hline Comprensión del & Configurar un plan \\
\hline problema & ¿Qué estrategias utilizarías \\
\hline $\begin{array}{l}\text { ¿Qué es una cara? ¿Qué } \\
\text { significan las huellas de }\end{array}$ & $\begin{array}{l}\text { para describir las huellas de } \\
\text { los sólidos? }\end{array}$ \\
\hline los cuerpos geométricos? & \\
\hline ¿Qué es plasmar? ¿Qué & huellas de los sólidos? \\
\hline $\begin{array}{l}\text { entiendes por superficie } \\
\text { plana? }\end{array}$ & $\begin{array}{l}¿ Q u e ́ \text { criterios se deben tener } \\
\text { en cuenta? }\end{array}$ \\
\hline $\begin{array}{l}\text { ¿Con qué cuerpo } \\
\text { geométrico estás } \\
\text { trabajando?; ¿cómo } \\
\text { son las caras del cubo?; } \\
\text { ¿cómo es el cono?, } \\
\text { ¿cómo son sus caras o su } \\
\text { superficie?, ¿qué forma } \\
\text { tiene la parte sobre la que } \\
\text { se apoya?, ¿qué nombre } \\
\text { recibe esa parte? }\end{array}$ & $\begin{array}{l}\text { ¿Qué características } \\
\text { encontraste en la figura? }\end{array}$ \\
\hline
\end{tabular}




\begin{tabular}{cccl}
$\begin{array}{c}\text { Nivel de } \\
\text { desempeño }\end{array}$ & Escala & $\begin{array}{c}\boldsymbol{N}^{\circ} \text { de } \\
\text { preguntas }\end{array}$ & Porcentaje \\
Satisfactorio & $\begin{array}{l}316-399 \\
\text { puntos }\end{array}$ & $14-17$ & $\begin{array}{l}63.6 \% \\
<80 \%\end{array}$ \\
Avanzado & $\begin{array}{l}400-500 \\
\text { puntos }\end{array}$ & $18-22$ & $>=80 \%$ \\
\hline
\end{tabular}

Fuente: Adaptación de Guía de Interpretación y Uso de Resultados de las pruebas SABER $3^{\circ}, 5^{\circ}$ y $9^{\circ}$. Establecimientos Educativos. Colombia 2015. Versión 1.

Las formas geométrica plasmadas en la hoja de papel desde la parte superior e inferior son iguales?

Manos a la obra con la estrategia seleccionada.

$¿$ Qué caracteriza a la figura geométrica plasmada desde la superior e inferior en un prisma?, ¿en un cubo?, ¿en una pirámide?, ¿en el cilindro?

¿Qué figura es la base de un: cubo, cilindro, pirámide y cono?

Por otro lado, con la metodología de trabajo cooperativo se atendió a desarrollar habilidades sociales y de pensamiento como son: el respeto por el uso de la palabra, parafrasear, preguntar por justificación y razones, pedir ayuda para aclaraciones, ayudar al compañero y criticar ideas, no personas, con el propósito de propiciar mejores ambientes de aprendizaje en los estudiantes.

\section{ANÁLISIS DE RESULTADOS}

Se presenta el análisis de los resultados obtenidos por medio del cálculo de algunas medidas de tendencia central y niveles de significancia que se realiza en función de los objetivos de investigación.

Para realizar la clasificación de los niveles de desempeño de los estudiantes se tuvo en cuenta ladistribución porcentual utilizada por el ICFES para su clasificación (ver Tabla 4), de acuerdo con el número de preguntas correctas a nivel general (22 preguntas), distribuidas por competencias.

Tabla 4. Distribución porcentual de los niveles de desempeño

$\begin{array}{cccc}\begin{array}{c}\text { Nivel de } \\ \text { desempeño }\end{array} & \text { Escala } & \begin{array}{c}\text { No de } \\ \text { preguntas }\end{array} & \text { Porcentaje } \\ \text { Insuficiente } & \begin{array}{l}100-226 \\ \text { puntos }\end{array} & 0-9 & <45.4 \% \\ \text { Mínimo } & \begin{array}{l}227-315 \\ \text { puntos }\end{array} & 10-13 & \begin{array}{l}45.4 \%,< \\ 63.6 \%\end{array}\end{array}$

\section{ANálisis de RESULTados DEL PRETEST GC-GE}

Una vez analizados los resultados de las preguntas acertadas, el porcentaje de estudiantes ubicados en el nivel insuficiente para el grupo control (GC) y grupo experimental (GE) es 44\%, y 53\% respectivamente (Gráfica 1), es decir, los estudiantes presentan dificultad para solucionar situaciones problemas que requieran identificar patrones y regularidades en el uso y representación de los sólidos y reconocer las propiedades que son dejadas invariantes cuando se aplica una transformación en el plano.

En cuanto al nivel de desempeño mínimo, el 44\% y 21\% de los estudiantes de GC y GE respectivamente se encuentran ubicados en este nivel, es decir, solucionan situaciones problemas de menor complejidad relacionados con asociar desarrollos planos con los respectivos sólidos y descomponer en regiones parciales figuras planas y sólidos; mientras que el 14\% faltante del GC se encuentra situado en los niveles satisfactorio (11\%) y avanzado (3\%) y el $26 \%$ de los estudiantes del GE se encuentran distribuidos en estos dos niveles.

Gráfica 1. Nivel de desempeño pretest de los estudiantes del Grado 5º de la Institución Educativa Villa Cielo

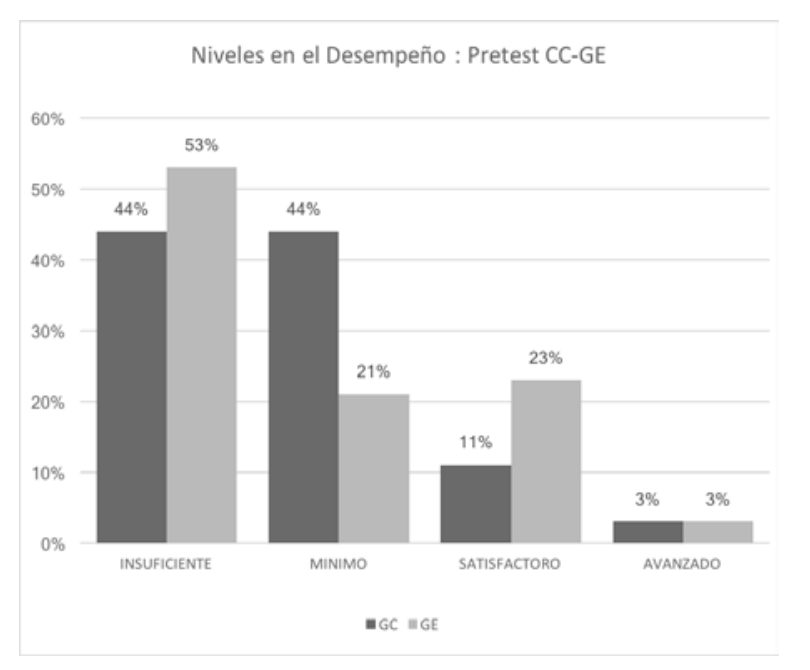

Fuente: elaboración propia.
Desarrollo de las competencias matemáticas en el pensamiento geométrico, a través del método heurístico de Polya
| Panorama | pp. 53-67 | Volumen 11 | Número 21 | Julio-Diciembre | 2017 
María Patiño

Garcés |

Eliana Sáenz Mass |

Juana Robles González |
De acuerdo con los resultados obtenidos (Gráfica 2) en la prueba pretest, el 36\% y $41 \%$ los estudiantes del GC y GE, respectivamente, alcanzaron a responder correctamente preguntas relacionadas con la competencia de razonamiento, es decir, representan, relacionan, construyen y descomponen figuras planas y sólidos a partir de condiciones dadas.

A su vez, en la competencia de comunicación, aproximadamente el $61 \%$ de los estudiantes en cada uno de los grupos no solucionan situaciones problemas, que corresponden a identificar, interpretar y describir procedimientos para la construcción y descomposición de figuras y objetos. Como puede observarse en la Gráfica 2, el promedio de respuestas correctas fue de $30 \%$.

Con respecto a la competencia de resolución de problemas, en esta se evidencia que alrededor del $62 \%$ de los estudiantes no solucionan problemas que requieran identificar patrones y regularidades usando representaciones geométricas.

Gráfica 2. Porcentajes de las competencias en el pretest en cada uno de los grupos

\section{Competencias en el Pretest: GC-GE}

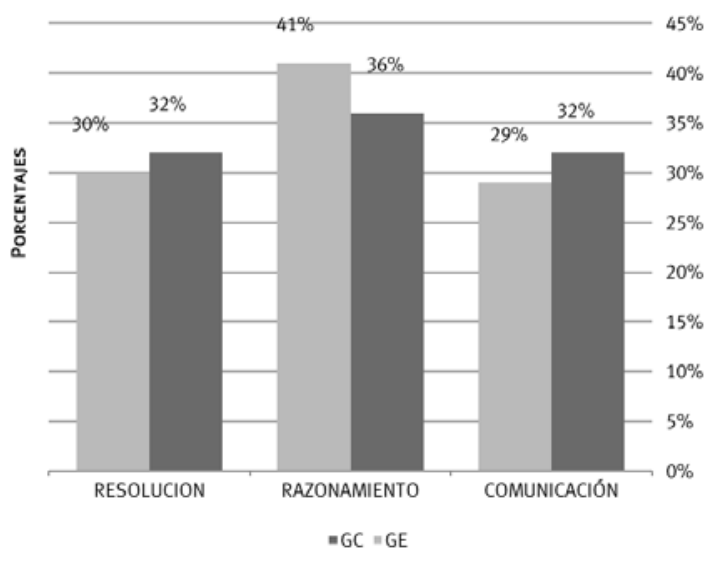

Fuente: elaboración propia.

\section{ANÁLISIS DE RESULTADOS POSTEST GC-CE}

En el análisis realizado a las preguntas acertadas por los estudiantes en el postest (Gráfica 3), se observa que en grupo control aproximadamente $53 \%$ de los estudiantes se encuentran en el nivel insuficiente, es decir, no solucionan problemas de menor complejidad relacionados se encuentran estudiantes ubicados en este nivel, indicando cambios positivos en los desempeños de los estudiantes en el pensamiento geométrico en la unidad de estudio solidos geométricos.

Con respecto al nivel de desempeño satisfactorio y avanzado, el GE alcanza un 94\%, es decir, los estudiantes solucionan problemas de mayor complejidad, relacionado con la comparación de diferentes atributos de figuras y sólidos a partir de sus características y establecer relaciones entre ellos.

Gráfica 3. Niveles de desempeño de los estudiantes de los grados $5^{\circ}$ en el postest

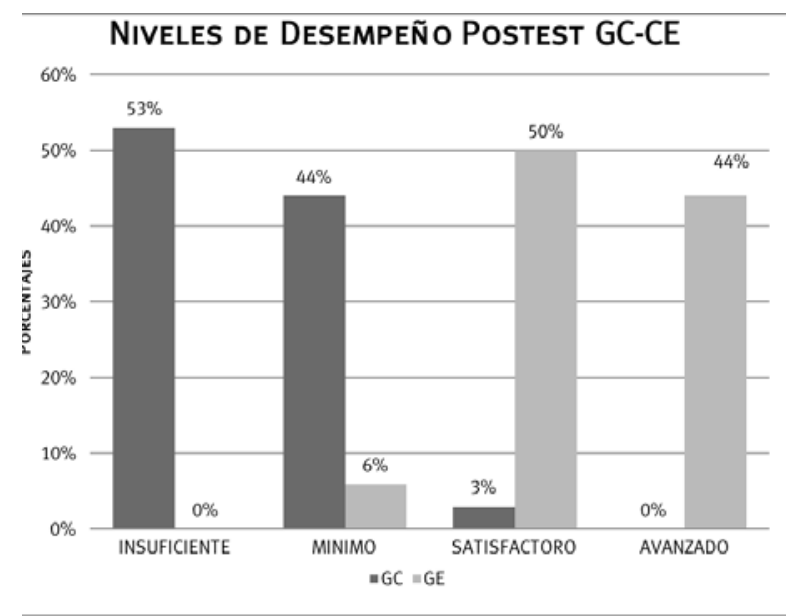

Fuente: elaboración propia.

Todo lo anterior confirma cambios positivos en la variable dependiente (pensamiento geométrico), demostrando así que la resolución de problemas desde el modelo de Polya con metodología de trabajo cooperativo es una estrategia eficaz, que servirá de apoyo a otros e investigadores para seguir profundizando en el conocimiento didáctico del contenido.

En la Tabla 5 se observa la estadística descriptiva para el GC y GE; en ella se evidencia diferencias en las medias después de la aplicación de la estrategia.

Tabla 5. Estadística descriptiva postest GC-GE

\begin{tabular}{ccccc}
\multicolumn{2}{c}{ GRUPO } & Media & $\begin{array}{c}\text { Des. } \\
\text { típica }\end{array}$ & $\begin{array}{c}\text { Error tip. } \\
\text { de la media }\end{array}$ \\
Pos-Raz. & GC & 3,68 & 1,319 &, 226 \\
\cline { 2 - 5 } & GE & 6,24 &, 955 &, 164 \\
\hline
\end{tabular}




\begin{tabular}{ccccc} 
GRUPO & Media & $\begin{array}{c}\text { Des. } \\
\text { típica }\end{array}$ & $\begin{array}{c}\text { Error tip. } \\
\text { de la media }\end{array}$ \\
Pos-Com. & GC & 2,82 & 1,218 &, 209 \\
\cline { 2 - 5 } & GE & 5,50 & 1,619 &, 278 \\
\hline \multirow{2}{*}{ Pos-Res. } & GC & 2,38 & 1,155 &, 198 \\
\cline { 2 - 5 } & GE & 5,09 & 1,055 &, 181 \\
\hline \multirow{2}{*}{ Pos-Total. } & GC & 8,94 & 2,361 &, 405 \\
\cline { 2 - 5 } & GE & 16,82 & 2,302 &, 395 \\
\hline
\end{tabular}

Fuente: SPSS 21. Versión libre.

Haciendo un análisis de los grupos, en la Tabla 3 se observan cambios en las medias en GC y el GE, lo cual nos sirvió de soporte al encontrar diferencias entre los grupos, es decir, los estudiantes del GE solucionaron situaciones problemas relacionadas con asociar desarrollos planos y sus respectivos sólidos, así como descomponer en regiones parciales figuras planas y sólidos, lo cual indica que la estrategia del método de las heurísticas de Polya fue eficaz.

Por otro lado, para determinar las características de la población se utilizó la prueba de Levene (Tabla 6). En ella se observa que los grupos son homogéneos dentro de cada uno ( $p>0.01)$, también se evidencia que el desempeño en cada una de las competencias y a nivel general entre el GC y GE es diferente ( $\mathrm{p}<0.05)$, lo cual está indicando desempeños distintos después del tiempo de la aplicación de la estrategia, presentándose una favorabilidad en el GE donde los promedios aumentaron significativamente como puede observarse en la Tabla 2.

Tabla 6. Prueba T por comparación de las competencias GC-GE
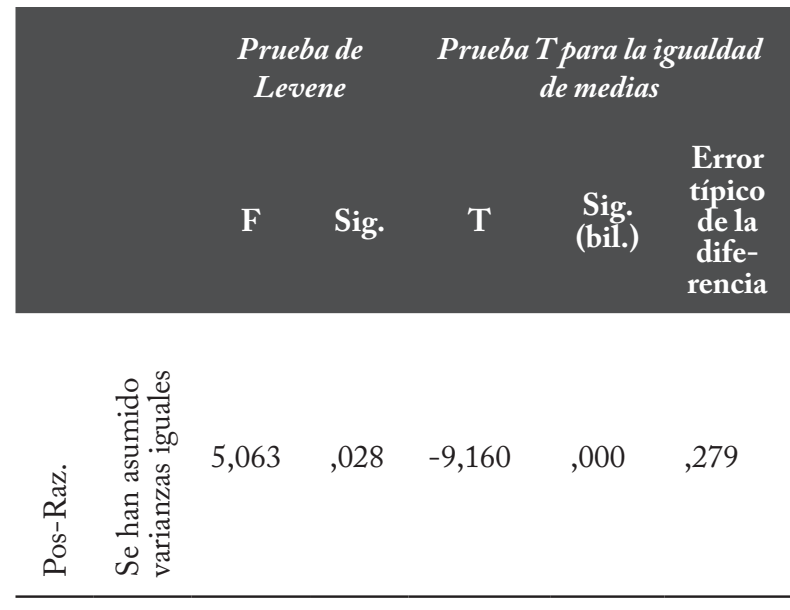

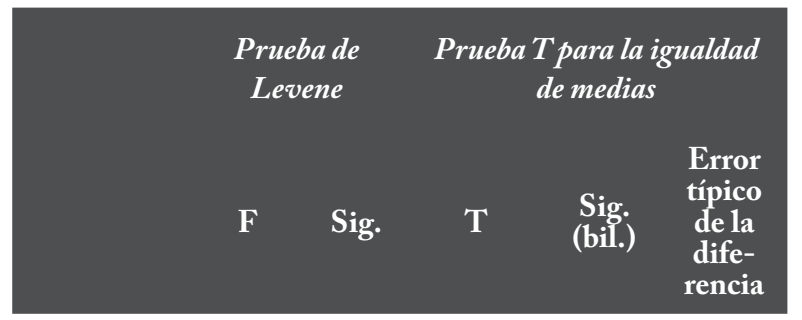

Desarrollo de las competencias matemáticas en el pensamiento geométrico, a través del método

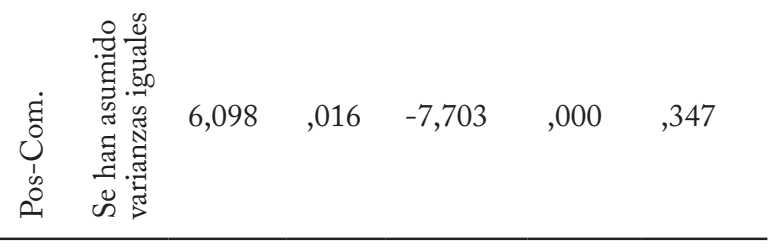
heurístico de Polya

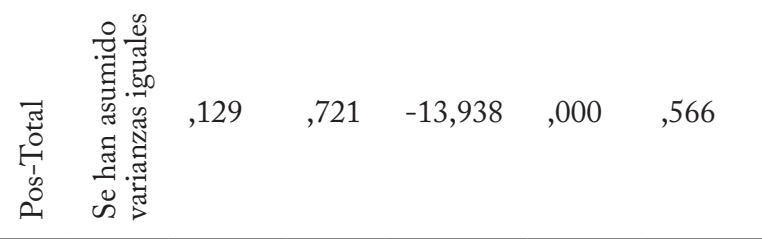

Fuente: SPSS 21. Versión libre.

\section{ANÁLISIS DE RESULTADOS PRETEST-POSTEST GC}

En la Tabla 7 se observa la estadística descriptiva para este grupo, observando los cambios en las medias antes y después de un tiempo determinado (tres meses).

Tabla 7. Muestra relacionada pretest - postest GC

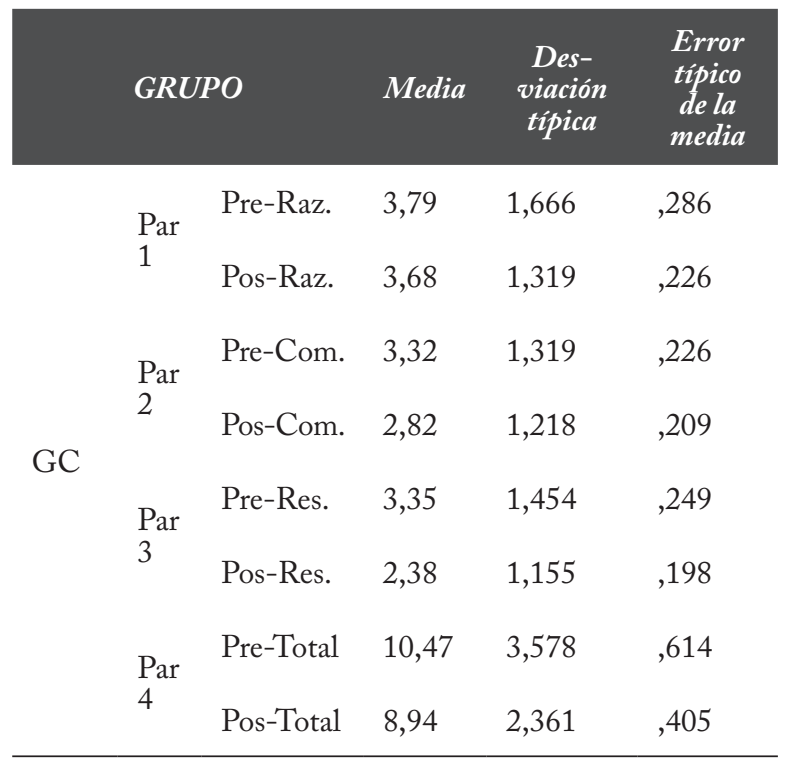

| Panorama | pp. 53-67 | Volumen 11 | Número 21 | Julio-Diciembre | 2017 
María Patiño

Garcés |

Eliana Sáenz Mass |

Juana Robles

González |

Por otro lado, la Tabla 8 indica que no hubo diferencia para este grupo en las competencias razonamiento y comunicación ( $p>0,05)$, en contraste con la competencia resolución donde se observa diferencias en el antes y después, situación que se pudo originar debido a las estrategias utilizadas por el docente, la falta de innovación de los recursos y materiales que propicien autonomía e interés en el estudiante para aprender, la falta de exploración de conocimientos previos de estudiantes, coincidiendo con lo descrito por Lastra (2005).

Tabla 8. Muestra relacionada pretest - postest GC
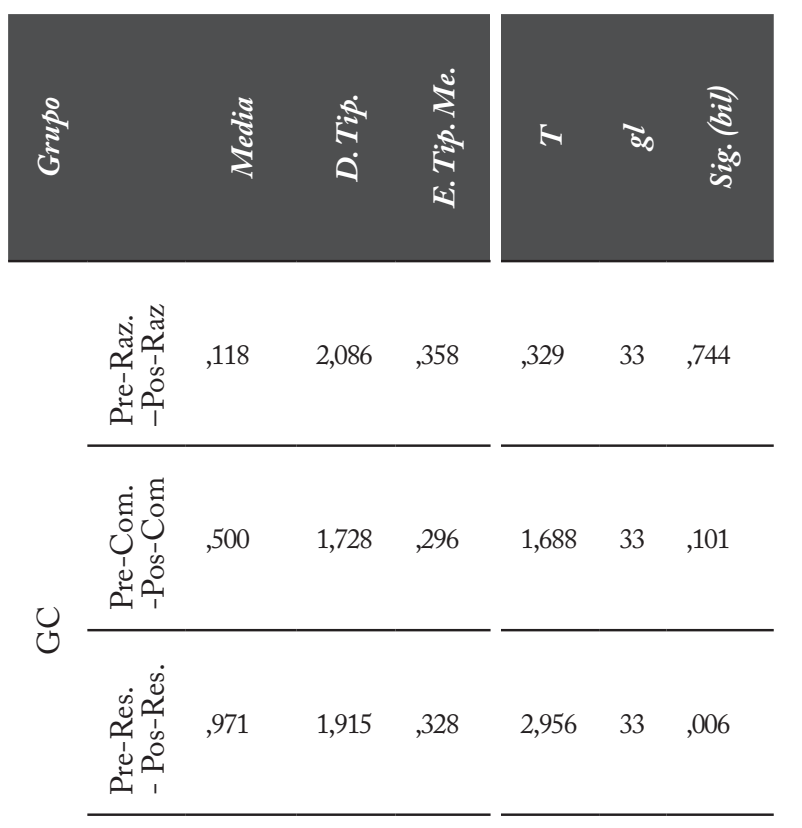

Panorama pp. 53-67|

Volumen 11 |

Número 21 |

Julio-Diciembre | 2017 |

Fuente: SPSS 21. Versión Libre.

\section{PRETEST - POSTEST GE}

La estadística descriptiva del GE se puede observar en la Tabla 9, el valor de las medias para identificar el comportamiento de las competencias en el antes y después de la intervención.

\begin{tabular}{|c|c|c|c|c|c|}
\hline & \multicolumn{2}{|c|}{ GRUPO } & Media & $\begin{array}{l}\text { Des. } \\
\text { típico }\end{array}$ & $\begin{array}{l}\text { Error } \\
\text { tip me }\end{array}$ \\
\hline \multirow{8}{*}{ GE } & \multirow{2}{*}{ Par 1} & Pre-Raz. & 4,35 & 1,228 & ,211 \\
\hline & & Pos-Raz. & 6,24 & ,955 & ,164 \\
\hline & \multirow[b]{2}{*}{ Par 2} & Pre-Com. & 2,97 & 1,381 & ,237 \\
\hline & & $\begin{array}{l}\text { Pos- } \\
\text { Com. }\end{array}$ & 5,50 & 1,619 & ,278 \\
\hline & \multirow{2}{*}{ Par 3} & Pre-Res. & 3,12 & 1,493 & ,256 \\
\hline & & Pos-Res. & 5,09 & 1,055 & 181 \\
\hline & \multirow{2}{*}{ Par 4} & Pre-Total & 10,44 & 2,776 & ,476 \\
\hline & & Pos-Total & 16,82 & 2,302 & ,395 \\
\hline
\end{tabular}

Fuente: SPSS 21. Versión libre.

Por otro lado, la Tabla 10 indica cambios $(\mathrm{p}<0.05)$ en los desempeños de los estudiantes en cada una de las competencias, es decir, los estudiantes solucionan situaciones problemas de mayor complejidad relacionadas con los sólidos geométricos.

\section{DISCUSIÓN}

En este marco se asume un modelo centrado en la resolución de problemas, con la intención de promover estrategias didácticas a partir del planteamiento de situaciones problema con el fin de desarrollar competencias que propicien aprendizajes significativos en los estudiantes. Debido a este planteamiento se estudia la eficacia de la estrategia didáctica del método heurístico de Polya para desarrollar las competencias de razonamiento, comunicación y resolución de problemas en el pensamiento geométrico.

Al observar los resultados del postest se encuentra concordancia con los obtenidos en sus investigaciones por autores como Boscan y Klever (2012), Aguilar y Navarro (2012), quienes concluyeron que la resolución de problemas según Polya favorece los aprendizajes de los estudiantes.

En este orden de ideas, en cuanto al desarrollo de competencias del pensamiento geométrico, en la unidad de aprendizaje sólidos, este estudio ratifica lo expuesto por Guillén (2010) y Osorno (2014), cuando resaltan la importancia del reconocimiento de los sólidos para 


\begin{tabular}{|c|c|c|c|c|c|c|c|c|c|}
\hline & \multirow[b]{2}{*}{ Grupo } & \multirow[b]{2}{*}{ Media } & \multirow[b]{2}{*}{ Des. } & \multicolumn{3}{|c|}{$95 \%$ I conf diferencia } & \multirow[b]{2}{*}{$T$} & \multirow[b]{2}{*}{$G l$} & \multirow[b]{2}{*}{ Sig. (bil) } \\
\hline & & & & $\begin{array}{c}E . T \text { de la } \\
\text { media }\end{array}$ & Inf. & Sup. & & & \\
\hline \multirow{4}{*}{ 師 } & $\begin{array}{l}\text { Pre-Raz. } \\
\text {-Pos-Raz. }\end{array}$ & $-1,882$ & 1,552 & ,266 & $-2,424$ & $-1,341$ & $-7,070$ & 33 & ,000 \\
\hline & $\begin{array}{l}\text { Pre-Com.- } \\
\text { Pos-Com }\end{array}$ & $-2,529$ & 2,149 & ,369 & $-3,279$ & $-1,779$ & $-6,862$ & 33 & ,000 \\
\hline & $\begin{array}{l}\text { Pre-Res } \\
\text { - Pos-Res }\end{array}$ & $-1,971$ & 1,586 & ,272 & $-2,524$ & $-1,417$ & $-7,247$ & 33 &, 000 \\
\hline & $\begin{array}{l}\text { Pre-Total } \\
\text { - Pos-Total }\end{array}$ & $-6,382$ & 2,850 & ,489 & $-7,377$ & $-5,388$ & $-13,058$ & 33 & ,000 \\
\hline
\end{tabular}

$-5,388$

desarrollar el pensamiento geométrico, y con respecto a lo expuesto por Blanco (2013), al destacar la influencia de lo didáctico y social para la comprensión de la geometría espacial.

Por otro lado, al aplicar la metodología de trabajo cooperativo en uno de los grupos experimental, los resultados después de la intervención se asemejan a los encontrados en las investigaciones de los autores Ferreiro (2007) y González y García (2007), quienes sostienen que el aprendizaje cooperativo incrementa y diversifica las habilidades comunicativas (comprender, explicar, preguntar y responder), utilizando un lenguaje apropiado, favoreciendo los aprendizajes de los estudiantes.

La valoración de las pruebas realizadas antes y después de la intervención para evaluar la efectividad de la estrategia didáctica a la luz del objetivo general se determina mediante pruebas estadísticas. En ellas se encontró que en el pretest no había diferencias significativas en el desempeño de los estudiantes en cada una de las competencias, mientras que el postest hay diferencias significativas entre los grupos, presentándose favorabilidad en el grupo experimental, concluyendo que el método heurístico de Polya desarrolla en los estudiantes habilidades y destrezas para clasificar, interpretar y resolver situaciones problemas relacionados con la geometría y otros campos. De esta manera se comprueba lo dicho por Polya (1981), quien afirma que solucionar un problema es crear habilidades posteriores para resolver cualquier tipo de problema.

En cuanto al GE, los estudiantes presentan mejores desempeños al solucionar situaciones problema al comparar, identificar y representar objetos tridimensionales, debido a la interacción de manera responsable y constructiva de todos los miembros del equipo de trabajo para comunicar con claridad y fluidez, ya sea por escrito y oral los resultados del problema coincidiendo con lo expuesto por Barnett et al. (2003).

Lo anterior refleja que la intervención didáctica alcanzó resultados positivos en el desarrollo de las competencias en el GE, donde se observan avances importantes y satisfactorios en el nivel de sus desempeños, lo que indica que los estudiantes superaron dificultades presentadas y accedieron a un nivel más avanzado, dando solución a situaciones que requieren un orden más complejo.

A partir de esto se puede suponer que las actividades propuestas y el desarrollo de las sesiones de trabajo representaron una oportunidad para desarrollar las competencias matemáticas, fortaleciendo en los estudiantes procesos propios de cada una de ellas.
| Panorama | pp. 53-67 | Volumen 11 | Número 21 | Julio-Diciembre | 2017 


\section{CONCLUSIONES}

Los resultados obtenidos en la investigación han permitido verificar los objetivos planteados al inicio del estudio y extraer algunas conclusiones respecto a la incidencia de la aplicación de la intervención didáctica diseñada y aplicada, teniendo en cuenta el método heurístico de Polya (1981) para el desarrollo del pensamiento geométrico; recapitulando, se encontró que:

Juana Robles

González |

Para la aplicación de la intervención del método heurístico de Polya se realizó un pretest, identificando las debilidades de los estudiantes y a partir del análisis de estas se diseñó y aplicó unas guías de aprendizaje basado en el método heurístico de Polya en el tema de los sólidos geométricos. En el desarrollo de cada una de las actividades de aprendizaje se evidenció que los estudiantes, en su interactuar con ellos mismos y el entorno social, favorecen la construcción de los conceptos obteniendo así mejoras en el aprendizaje, coincidiendo con lo expuesto por Barnett et al., (2003).

Después de realizar la intervención con el método heurístico de Polya, el nivel de desempeño en las competencias de razonamiento, comunicación y resolución de problemas aumentó satisfactoriamente en el GE, lo que indica la efectividad de la propuesta.

En relación con el GE, después de realizar la intervención se evidencia que la metodología de trabajo cooperativo fortalece aún más la competencia de comunicación, debido a que en esta estrategia se pretende desarrollar habilidades que propenden por la construcción colectiva de los aprendizajes.

Del mismo modo, con la ayuda de la estrategia didáctica del método heurístico de Polya y el trabajo cooperativo se pudo comprender la dinámica del grupo en el

Panorama | pp. 53-67| Volumen 11 | Número 21 | Julio-Diciembre | conocimiento, como una herramienta útil para la labor docente, además convertirse en un referente para otras investigaciones.
La implementación de la estrategia didáctica en un periodo académico sirvió para mejorar las habilidades y competencias del pensamiento geométrico en los grupos experimentales, donde los aprendizajes de los estudiantes fueron significativos al sentirse parte activa del proceso, expresando sus pensamientos y ayudando a la construcción del conocimiento. En este sentido, el docente orienta y acompaña el trabajo didáctico.

La resolución de problemas puede emplearse exitosamente como una estrategia para desarrollar las competencias a través de las etapas de resolución de problemas desde el método heurístico de Polya (1981), adaptándolas de manera que respondan a los contextos actuales y a las necesidades educativas propias de la población.

En la parte curricular, considerar la posibilidad de incluir en los planes de área la estrategia didáctica del método heurístico de Polya y la metodología de trabajo cooperativo para desarrollar las unidades didácticas, que al momento de ser aplicadas de manera responsable y no improvisada favorecen las prácticas pedagógicas y el aprendizaje de los estudiantes.

Finalmente, se logró constatar también que la estrategia didáctica utilizada en esta investigación realmente era necesaria para conocer y solucionar problemas relacionados con objetos matemáticos, directamente con la geometría y de esta manera conseguir que los estudiantes de la Institución Educativa Villa Cielo, del municipio de Montería, cuenten con una propuesta curricular completa que tenga como base el método heurístico de Polya.

\section{REFERENCIAS}

1. Aguilar, M. (2000). Aplicación de una estrategia de resolución de problemas matemáticos en niños. Psicol. Gral y Aplic, Departamento de Psicología de la Universidad de Cádiz.

2. Arnau, J. (1995). Diseño de Investigación Aplicada. Barcelona: Universitat Oberta de Cataluña.

3. Báez, R. e Iglesias, M. (2007). Principios didácticos a seguir en el proceso enseñanza y aprendizaje de la geometría en la UPEL "E1 Mácaro". Enseñanza de la Matemática, 67-87.

4. Blanco, H. (2013). Representaciones gráficas de cuerpos geométricos. Un análisis de los cuerpos a través de sus representaciones. Mexico: Instituto Politécnico Nacional. 
5. Boscán, M. y Klever, K. (2012). Metodología basada en el método heurístico de Polya para el aprendizaje de resolución de problemas matemáticos. Universidad Simón Bolívar.

6. Campbell, D. y Stanley, J. (1995). Diseños experimentales y cuasiexperimentales en la investigación social. Buenos Aires: Amorrortu Editores S.A.

7. Elliott, J. (1996). El cambio educativo desde la investigación-acción. Madrid: Morata.

8. Ferreiro, R. y Calderón, M. (2001). El ABC del aprendizaje cooperativo. Trabajo en equipo para enseñar y aprender. México D.F.: Trillas.

9. González, E. y Guillén, G. (2006). La enseñanza de la geometría en la Educación Primaria. Enseñanzalaprendizaje de la geometría en la formación de profesores de primaria a la enseñanza de esta materia en el aula: estudio de casos. España: Departamento de Didactica de la Matemática. Universidad de Valencia.

10. Guillén, G.. (2010). ¿̇Por qué usar los sólidos como contexto en la enseñanzal aprendizaje de la geometría? $\dot{\zeta}$ en la investigación? España: Universidad de Valencia.

11. Gutierrez, A. (1998). Las representaciones planas de cuerpos 3-dimensionales en la enseñanza de la geometría espacial. Revista EMA, 193-220.

12. Hernández, V.y Villalba, M. (Trad.). (2001). Perspectiva en la enseñanza de la geometría para el siglo XXI. Recuperado de: http://www.euclides. org/menu/articles/article2.htm

13. Ibarra, A. (2012). La enseñanza de la resolución de problemas matemáticos en la escuela primaria: experiencia de los profesores. Jalisco, México: Trabajo de Tesis.

14. ICFES. (2015). Guía de interpretación y uso de resultados de las pruebas $\mathrm{SABER} 3^{\circ}, 5^{\circ}$ y $9^{\circ}$. Guí, 93-95.

15. Iriarte,A. (2011). Desarrollo de las competencias en solución de problemas desde una didáctica con enfoque metacognitivo. Sincelejo: SUE Caribe. Universidad de Sucre.

16. Johnson, D.,Johnson, R. y Holubec, E. (1994) Aprendizaje cooperativo en el aula. Virginia: Association For Supervision and Curriculum Development.

17. Kuder, G. (1937). The theory of estimation of test reliablity. Psychometrika (2).

18. Lastra, S. (2005). Propuesta Metodológica de Enseñanza y Aprendizaje de la Geometría, aplicada en Escuelas Criticas. Chile: Universidad de Chile.
19. López, O. y García, S. (2008). La enseñanza de la geometría. México, D.F.: INEE.

20. MEN.(1998 - 2016 ). Referentes Curriculares de Matemáticas. Bogotá: Ministerio de Educación Nacional.

21. MEN. (1998). Lineamientos Curriculares. Bogotá: Ministerio de Educación Nacional.

22. MEN. (2006). Estándares Básicos de Competencias en Matemáticas. Bogotá: Ministerio de Educación Nacional.

23. MEN. (2006). Lineamientos Curriculares. Bogotá: Ministerio de Educación Nacional.

24. OCDE. (2015). El Programa PISA de la OCDE. Qué es y para qué sirve. París.

25. OCDE. (2014). Resultados de PISA 2012 en Foco.

26. Paredes, Z., Iglesias, M. y Ortiz, J. (2007). Sistemas de cálculo simbólico y resolución de problemas en la formación inicial de docente. REICE, Revista Iberoamericana sobre Calidad, Eficacia y Cambio en Educación. Recuperado de: https://revistas.uam.es/index.php/reice/article/ view/5425

27. Pérez, A. (2007). La naturaleza de las competencias básicas y sus aplicaciones pedagógicas. Cantabria.

28. Polya, G. (1981). Cómo plantear y resolver problemas. México:Trillas.

29. Polya, G. (1981). Mathematical Discovery. New York: Wiley \& Sons, Inc.

30. Rojas, J. (2014). Estrategia didáctica para la enseñanza de la geometría del hexaedro. Medellin: Universidad Nacional de Colombia.

31. Schoenfeld, A. (1985). Mathematical problem solving. Orlando, Florida, EE.UU.: Academic Press.

32. Tobón, S. (2006). Aspectos Básicos de la Formación Basada en Competencias. Proyecto Mesesup. Telca.

33. Velázquez, C. (2013). Análisis de la implementación de aprendizaje cooperativo durante la escolarización obligatoria en el área de educación física. Valladolid:Tesis doctoral.

34.

\section{Desarrollo de las competencias \\ matemáticas en \\ el pensamiento \\ geométrico, \\ a través \\ del método \\ heurístico de \\ Polya}

| Panorama

| pp. 53-67

Volumen 11

| Número 21

| Julio-Diciembre 
María Patiño

Garcés |

Eliana Sáenz

Mass |

Juana Robles

González |

Panorama |

pp. 53-67|

Volumen 11 |

Número 21 |

Julio-Diciembre |

2017 |
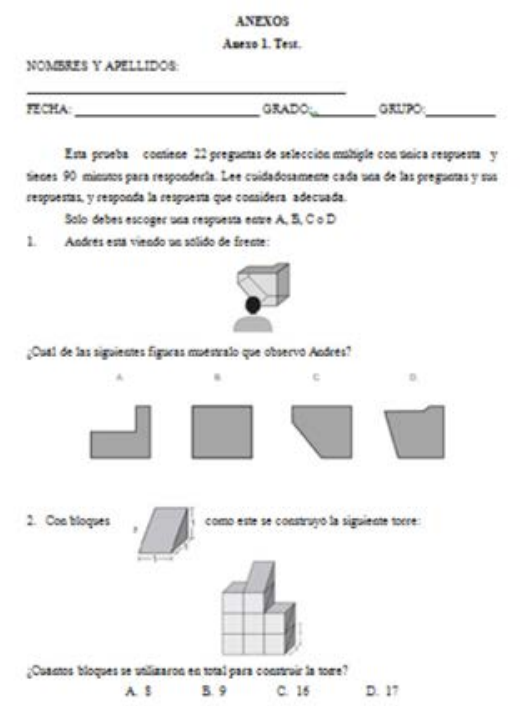

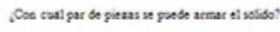

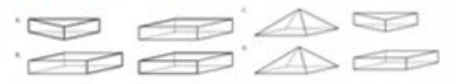

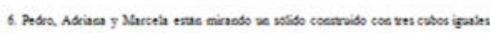

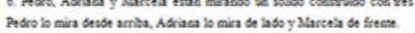
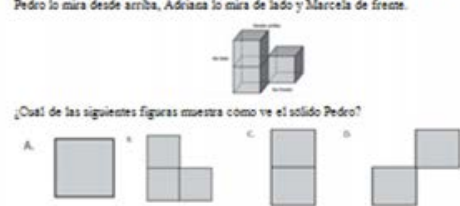

7. Obuera ia ware of ia figea 1
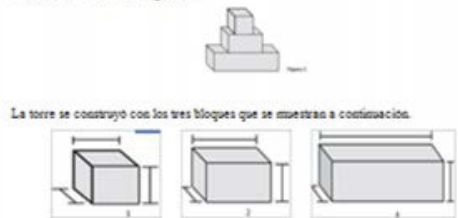

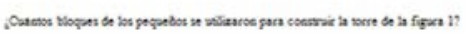

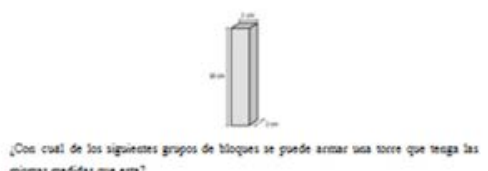

$$
\text { macas mosisas pa esa? }
$$

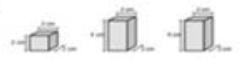

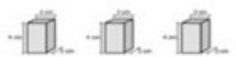

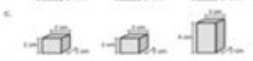

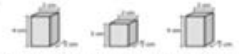

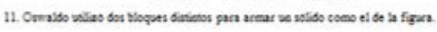

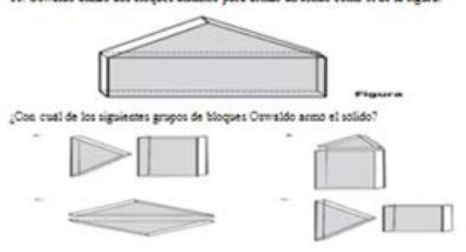

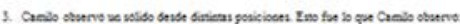
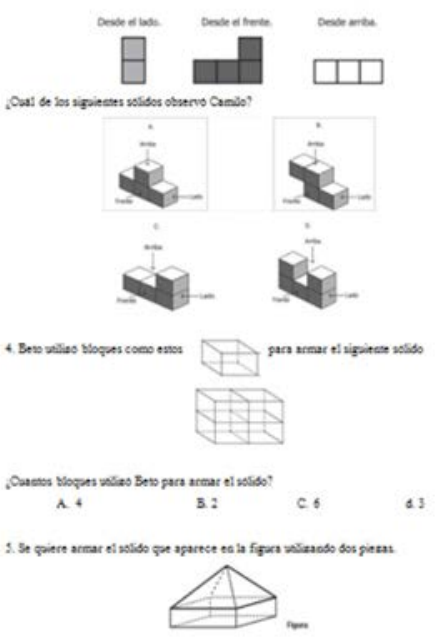

A. $3 . \quad$ C. $\quad$ D. 13

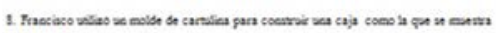
es a tiges
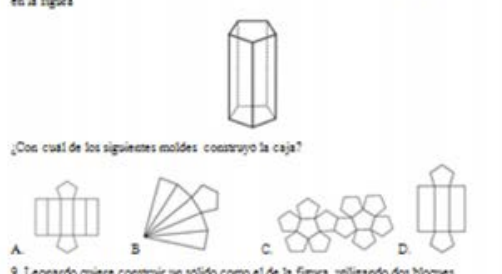

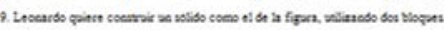

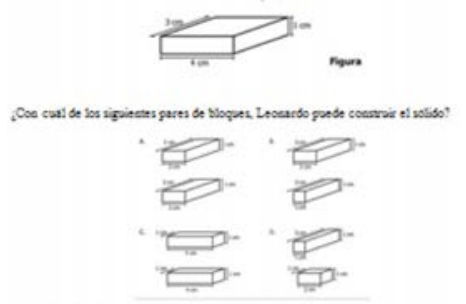

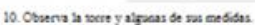

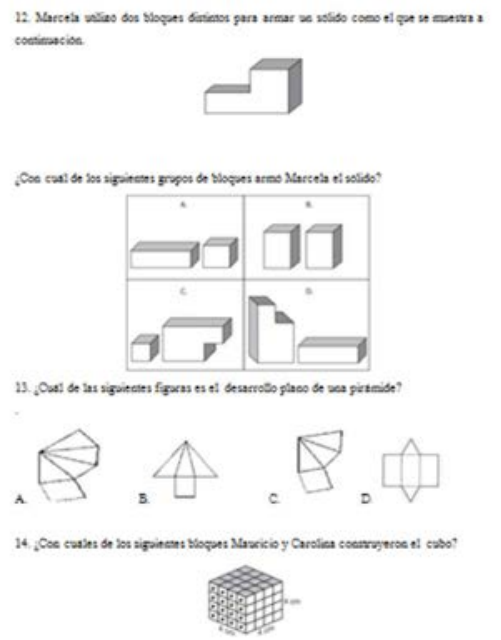


